\title{
Combinatorial Landscape Analysis for $k$-SAT Instances
}

\author{
Andreas A. Albrecht, Peter C.R. Lane and Kathleen Steinhöfel
}

\begin{abstract}
Over the past ten years, methods from statistical physics have provided a deeper inside into the average complexity of hard combinatorial problems, culminating in a rigorous proof for the asymptotic behaviour of the $k$-SAT phase transition threshold by Achlioptas and Peres in 2004. On the other hand, when dealing with individual instances of hard problems, gathering information about specific properties of instances in a pre-processing phase might be helpful for an appropriate adjustment of local search-based procedures. In the present paper, we address both issues in the context of landscapes induced by $k$-SAT instances: Firstly, we utilize a sampling method devised by Garnier and Kallel in 2002 for approximations of the number of local maxima in landscapes generated by individual $k$-SAT instances and a simple neighbourhood relation. The objective function is given by the number of satisfied clauses. Secondly, we outline a method for obtaining upper bounds for the average number of local maxima in $k$-SAT instances which indicates some kind of phase transition for the neighbourhood-specific ratio $m / n=\Theta\left(2^{k} / k\right)$.
\end{abstract}

\section{INTRODUCTION}

$\mathbf{M}$ $\mathrm{UCH}$ attention has been paid in recent years to local search algorithms as one of the basic methods to solve $k$-SAT problems. A first summary was presented in [8] along with an empirical analysis of run-time distributions for various local search-based methods such as WalkSAT [22]. Improvements on run-time estimations for $k$-SAT problems as well as for CNFs with unconstrained clause lengths are reported in [3], [4], [6], [14], [17], [18], which are partly based on randomised local search methods. Significant progress has been achieved in the analysis of phase transitions since this effect was reported in [11], [23]. Sophisticated methods from statistical mechanics [12], [10], [13] provided quite accurate estimates for the crucial phase transition parameter, which eventually led to a rigorous proof of a tight bound of $2^{k} \cdot \log 2-O(k)$ for the phase transition threshold as presented in [1] (for an overview on statistical mechanics applied to combinatorial optimization we refer the reader to [9]).

In the present paper, we attempt to analyse the number of local maxima in a combinatorial landscape induced by a $k$ $\mathrm{CNF}$ and a simple neighbourhood function, with the objective function being the number of satisfied clauses for a given assignment of binary values. In recent years, combinatorial landscape analysis has become a major tool in the design of search-based algorithms, see [16]. For example, instancespecific landscape parameters such as the maximum value of the minimum escape height from local minima can be

A.A. Albrecht and P.C.R. Lane are with the School of Computer Science, University of Hertfordshire, Hatfield, Herts AL10 9AB, UK (email: \{A.Albrecht, P.C.Lane\}@ herts.ac.uk); K. Steinhöfel is with the Department of Computer Science, King's College London, London WC2R 2LS, UK (email: Kathleen.Steinhofel@kcl.ac.uk).

This work was partially supported by EPSRC Grant EP/D062012/1. utilized to obtain relatively tight bounds for the termination of local search when coupled with a confidence parameter, see [2]. The application of this type of run-time bounds to protein folding simulation exhibits a close correspondence between the simulation time (in number of transitions) and estimates of real folding times (in nanoseconds) of protein sequences [24], [25], which is due to the common source of thermodynamics (simulated annealing, minimizing free energy in protein foldings).

Reeves and Eremeev [15] have demonstrated how to incorporate the number of local optima into run-time estimates of local search algorithms. For landscapes that can be partitioned into attraction basins, they proved that with probability $\alpha$ all local optima have been covered by local search with random restart after a waiting time of $\nu \cdot \ln (\nu+\gamma)+z_{\alpha}$. $\sqrt{(\nu \cdot \pi)^{2} / 6+1-\nu \cdot \ln (\nu+\gamma)}$, where $\nu$ is the number of local optima, $\gamma$ is the Euler-Mascheroni constant, and $z_{\alpha}$ is an appropriate confidence coefficient. Thus, estimates for $\nu$ provide information, e.g., for the selection of the population size in parallelized versions of local search algorithms, such as genetic algorithms or evolutionary algorithms in general.

In a slightly different context (Max-SAT and local minima), Zhang [27] proposes a landscape-based method that performs especially well on overconstrained random MaxSAT instances. Moreover, Zhang's algorithm finds satisfiable solutions on large $k$-SAT instances more often than WalkSAT. The paper highlights the importance of how to deal with individual instances rather than with collections of (randomly selected) problem instances.

In our paper, we utilize the approach devised in [5] to estimate the number of local maxima for a given problem instance, where sample data are used to approximate a probability distribution associated with the landscape induced by the problem instance. The results are discussed against the information gathered by a complete analysis of the landscape for a limited number of $k$-SAT problem instances. Given the nature of the problem, we were able to analyse only small-scale instances and overall only a limited number of different, randomly generated $k$-SAT instances. Apart from the experimental analysis based on the Garnier/Kallelapproach, we derive a rough estimate of the average number of local maxima per $k$-CNF in terms of parameters of individual problem instances for the given, simple neighbourhood relation. The calculations indicate that for the ratio $m / n=$ $\Theta\left(2^{k} / k\right)$, where $m$ is the number of clauses and $n$ the number of variables, the magnitude of the average number of local maxima per $k$-CNF changes significantly. We note that the calculations depend on the type of the neighbourhood, i.e. other neighbourhood relations may produce different values, which will be subject of future research. 


\section{BASIC NOTATIONS}

We follow mainly the notations from [1]: for a set $V$ of $n$ Boolean variables let $C_{k}(V)$ denote the set of all $\left(\begin{array}{l}n \\ k\end{array}\right) \cdot 2^{k}$ different disjunctive $k$-clauses on $V$, i.e. repeated literals and tautologies are excluded. A $k$-CNF is formed by selecting $m$ different clauses $C$ from $C_{k}(V)$ and taking their conjunction. We note that the selection does not imply - as in [1] - that the $k$-CNF strictly depends upon all $n$ variables. The set of all such $k$-CNF consisting of $m$ clauses is denoted by $\mathcal{F}_{k}(n, m)$. The set of $m$ clauses forming $F \in \mathcal{F}_{k}(n, m)$ is denoted by $\mathcal{C}(F)$, and $\mathrm{Z}_{F}(\tilde{\sigma})$ is the number of satisfied clauses $C \in$ $\mathcal{C}(F)$ on the truth assignment $\tilde{\sigma}=\left(\sigma_{1}, \ldots, \sigma_{n}\right)$, i.e. $0 \leq$ $\mathcal{Z}_{F}(\tilde{\sigma}) \leq m$ and $F$ is satisfiable, if there exists $\tilde{\eta}$ such that $\mathcal{Z}_{F}(\tilde{\eta})=m$.

In [19], various neighbourhood functions are analysed that employ information about $\mathrm{Z}_{F}(\tilde{\sigma})$ and elements of $\mathcal{C}(F)$ that maximise changes of the objective function in one way or another. For example, flipping values of truth assignments is determined by unsatisfied clauses only, see also [21], [20]. We consider a simple, unconstrained (i.e., features of clauses w.r.t. $\mathrm{Z}_{F}(\tilde{\sigma})$ are not taken into account) neighbourhood function where the value of a single variable is flipped, which makes it possible to consider the elements of the unit cube $\{0,1\}^{n}$ as elements of the configuration space. Thus, the landscape $\mathcal{L}(F)$ for $F$ is induced by $\mathrm{Z}_{F}(\tilde{\sigma}), \tilde{\sigma} \in\{0,1\}^{n}$, and the neighbourhood relation

$$
\mathcal{N}(\tilde{\sigma})=\left\{\tilde{\sigma}^{\prime} \mid d\left(\tilde{\sigma}, \tilde{\sigma}^{\prime}\right)=1\right\},
$$

where $d\left(\tilde{\sigma}, \tilde{\sigma}^{\prime}\right)$ is the Hamming distance.

If $\forall \tilde{\sigma}^{\prime}\left(\tilde{\sigma}^{\prime} \in \mathcal{N}(\tilde{\sigma}) \rightarrow\left(\mathrm{Z}_{F}\left(\tilde{\sigma}^{\prime}\right) \leq \mathrm{Z}_{F}(\tilde{\sigma})\right)\right.$, then $\tilde{\sigma}$ is called a local maximum (which also covers global maxima).

\section{THE GARNIER/KALLEL-APPROACH}

In the present paper, we are solely concerned with the landscape analysis called inverse problem in [5], i.e. $M$ elements of the landscape are selected at random as initial points of a pre-defined local search procedure. Then, for $j$ initial points, where $1 \leq j \leq M$, the local search procedure is started and executed until a (local) maximum has been detected. The number of different (local) maxima is denoted by $\beta_{j}$. The local search procedure is quasi-deterministic and follows the steepest ascent rule: for the intermediate landscape element $\tilde{\sigma}$, all elements of $\mathcal{N}(\tilde{\sigma})$ are examined and one of the neighbours $\tilde{\sigma}^{\prime}$ with the highest value of $\mathrm{Z}_{F}\left(\tilde{\sigma}^{\prime}\right)$ among all neighbours is chosen as the successor of $\tilde{\sigma}$ in the search procedure. The search terminates, if no improvement of the objective function can be achieved. In [5], and the same applies to [15], a single element $\tilde{\sigma}^{\prime} \in \mathcal{N}(\tilde{\sigma})$ is assumed at each step that maximises $\mathrm{Z}_{F}\left(\tilde{\sigma}^{\prime}\right)$, which implies a partition of $\mathcal{L}$ into attraction basins $A_{i}$, where $1 \leq i \leq N$ for a total number of $N$ local and global maxima. The set $A_{i}$ consists of all elements of $\mathcal{L}$ that lead to the $i^{\text {th }}$ local or global maximum by the steepest ascent local search. The assumption affects the normalised size $\alpha_{i}=\left|A_{i}\right| /|\mathcal{L}|$ of attraction basins and $\sum_{i=1}^{N}\left|A_{i}\right| /|\mathcal{L}|=1$. Since we employ the Garnier/Kallelapproach in an experimental context, we assume in the following that the impact of random selections among $\tilde{\sigma}^{\prime}$ that maximise $Z_{F}\left(\tilde{\sigma}^{\prime}\right)$ within a given neighbourhood is negligible.

Garnier and Kallel (2002) assume that the normalised sizes $\alpha_{i}$ of attraction basins can be described by a distribution parametrized by some positive number $\gamma$ as follows: let $\left(Z_{i}\right)_{i=1, . ., N}$ be a sequence of independent random variables whose common distribution has density $p_{\gamma}$ defined by

$$
p_{\gamma}=\frac{\gamma^{\gamma}}{\Gamma(z)} \cdot z^{\gamma-1} \cdot e^{-\gamma \cdot z}
$$

where $\Gamma(z)=\int_{0}^{\infty} e^{-t} \cdot t^{z-1} d t$, i.e. (2) represents the Gamma distribution with the parameter setting $[\gamma, \gamma]$, see [5]. Let $H^{\gamma}$ denote the assumption that the $\left(\alpha_{i}\right)_{i=1, \ldots, N}$ can be approximated by $\left(Z_{i} / T_{N}\right)_{i=1, \ldots, N}$, where $T_{N}=\sum_{i=1}^{N} Z_{i}$ with each $Z_{i}$ having the density function $p_{\gamma}$. Furthermore, let $\beta_{j, \gamma}=\mathbb{E}_{\gamma}\left[\beta_{j}\right]$ denote the expected value of $\beta_{j}, j=1, \ldots, M$. Garnier and Kallel (2002) prove that

$$
\begin{aligned}
\beta_{j, \gamma}= & N \cdot\left(\begin{array}{c}
M \\
j
\end{array}\right) \cdot \frac{\Gamma(\gamma+j)}{\Gamma(\gamma)} \cdot \frac{\Gamma(N \cdot \gamma)}{\Gamma((N-1) \cdot \gamma)} \times \\
& \times \frac{\Gamma((N-1) \cdot \gamma+M-j)}{\Gamma(N \cdot \gamma+M)} .
\end{aligned}
$$

We note that for $N=M / r$, a fixed value of $M$, and appropriate approximations of the $\Gamma$-function, the $\beta_{j, \gamma}$ can be approximated according to (3) as functions of $(j, \gamma, r)$. For fixed $r$, Garnier and Kallel (2002) propose the $\chi^{2}$ test to approximate $\gamma$ for $H^{\gamma}$, which consists of calculating

$$
T_{\gamma}=\sum_{j=1}^{M} \frac{\left(\beta_{j}-\beta_{j, \gamma}\right)^{2}}{\beta_{j, \gamma}},
$$

where the $\beta_{j}$ are given from observation and the $\beta_{j, \gamma}$ are approximated according to (3). The goal is then to determine

$$
\gamma_{0}(r)=\operatorname{argmin}\left\{T_{\gamma}, \gamma>0\right\}
$$

by appropriate numerical methods. In our computational experiments, we incorporate the approximation of $\gamma_{0}(r)$ as a sub-routine in calculations where the parameter $r$ varies (is decremented) until $\gamma_{0}(r)$ changes only marginally for $r=r_{\text {appr }}$, see Section IV. Thus, for a fixed (but sufficiently large) value of $M$ the number of local maxima is finally estimated by

$$
N=\frac{M}{r_{\text {appr }}} .
$$

\section{COMPutational Experiments}

\section{A. Evaluation of random 3-SAT instances}

We fixed $k=3$ and for $n=14,20,21$ we randomly generated three to five instances from $\mathcal{F}_{3}(n, m)$ for varying ratios $m / n$ around the phase transion threshold $m / n \approx$ 4.267 .

For each of the $k$-CNF we executed a complete search for local/global maxima in $\{0,1\}^{14},\{0,1\}^{20}$ and $\{0,1\}^{21}$. The corresponding values of the number $N$ of maxima are shown in the second column of Table 1 and Table 2, respectively.

We then selected three values for $M$, the number of random points chosen in $\{0,1\}^{14},\{0,1\}^{20}$ and $\{0,1\}^{21}$ 
as initial elements for a deteministic steepest ascent search for local maxima. For each of the values $M_{i}, i=1,2,3$, and the natural order of the $M_{i}$ points we counted by $\beta_{j}^{i}$, $j=1, \ldots, M_{i}$ the number of different maxima detected by the first $j$ starting points for steepest ascent search. The values of $\beta_{M}^{i}$ are recorded in the fourth column of Table 1 and Table 2.

An example of one of the five 3 -CNF for $n=21$ is given below:

$\neg x_{0} \vee \neg x_{1} \vee \neg x_{8} \wedge \neg x_{0} \vee x_{2} \vee x_{15} \wedge \neg x_{0} \vee x_{2} \vee \neg x_{18} \wedge \neg x_{0} \vee x_{2} \vee$ $\neg x_{19} \wedge \neg x_{0} \vee \neg x_{3} \vee \neg x_{8} \wedge x_{0} \vee \neg x_{4} \vee \neg x_{10} \wedge \neg x_{0} \vee \neg x_{6} \vee \neg x_{14} \wedge$ $\neg x_{0} \vee \neg x_{9} \vee x_{16} \wedge \neg x_{0} \vee \neg x_{12} \vee x_{14} \wedge \neg x_{0} \vee \neg x_{12} \vee x_{17} \wedge x_{0} \vee$ $\neg x_{13} \vee \neg x_{18} \wedge x_{1} \vee \neg x_{2} \vee \neg x_{9} \wedge \neg x_{1} \vee \neg x_{2} \vee \neg x_{10} \wedge x_{1} \vee \neg x_{3} \vee$ $x_{4} \wedge \neg x_{1} \vee \neg x_{3} \vee \neg x_{14} \wedge x_{1} \vee \neg x_{3} \vee x_{20} \wedge \neg x_{1} \vee x_{4} \vee \neg x_{16} \wedge$ $x_{1} \vee \neg x_{5} \vee \neg x_{15} \wedge x_{1} \vee x_{5} \vee \neg x_{16} \wedge x_{1} \vee \neg x_{6} \vee \neg x_{11} \wedge x_{1} \vee \neg x_{6} \vee$ $\neg x_{17} \wedge x_{1} \vee x_{9} \vee x_{13} \wedge x_{1} \vee \neg x_{9} \vee \neg x_{15} \wedge \neg x_{1} \vee \neg x_{9} \vee x_{18} \wedge$ $\neg x_{1} \vee x_{12} \vee \neg x_{13} \wedge \neg x_{1} \vee \neg x_{14} \vee x_{17} \wedge \neg x_{1} \vee \neg x_{14} \vee \neg x_{18} \wedge$ $x_{1} \vee x_{17} \vee x_{18} \wedge \neg x_{2} \vee x_{3} \vee \neg x_{10} \wedge \neg x_{2} \vee x_{6} \vee x_{12} \wedge \neg x_{2} \vee x_{8} \vee$ $x_{16} \wedge \neg x_{2} \vee x_{11} \vee \neg x_{19} \wedge \neg x_{2} \vee x_{12} \vee \neg x_{15} \wedge \neg x_{2} \vee x_{14} \vee x_{15} \wedge$ $x_{2} \vee x_{14} \vee x_{16} \wedge x_{3} \vee \neg x_{4} \vee x_{7} \wedge \neg x_{3} \vee x_{6} \vee \neg x_{7} \wedge \neg x_{3} \vee \neg x_{8} \vee$ $x_{11} \wedge \neg x_{3} \vee x_{9} \vee x_{17} \wedge x_{3} \vee x_{10} \vee \neg x_{11} \wedge x_{3} \vee x_{11} \vee \neg x_{18} \wedge x_{3} \vee$ $x_{16} \vee x_{20} \wedge x_{4} \vee \neg x_{5} \vee \neg x_{13} \wedge x_{4} \vee x_{7} \vee \neg x_{11} \wedge x_{4} \vee x_{8} \vee \neg x_{12} \wedge$ $x_{4} \vee x_{13} \vee \neg x_{19} \wedge \neg x_{4} \vee x_{14} \vee \neg x_{19} \wedge x_{4} \vee x_{16} \vee x_{17} \wedge x_{5} \vee x_{6} \vee$ $\neg x_{19} \wedge \neg x_{5} \vee x_{7} \vee \neg x_{12} \wedge \neg x_{5} \vee \neg x_{7} \vee x_{16} \wedge \neg x_{5} \vee \neg x_{7} \vee x_{19} \wedge$ $x_{5} \vee x_{8} \vee x_{10} \wedge x_{5} \vee x_{8} \vee \neg x_{20} \wedge x_{5} \vee \neg x_{10} \vee x_{16} \wedge \neg x_{6} \vee x_{7} \vee$ $x_{8} \wedge \neg x_{6} \vee x_{7} \vee \neg x_{9} \wedge \neg x_{6} \vee x_{11} \vee x_{14} \wedge \neg x_{6} \vee \neg x_{12} \vee x_{14} \wedge x_{6} \vee$ $\neg x_{12} \vee x_{18} \wedge \neg x_{6} \vee \neg x_{12} \vee x_{20} \wedge \neg x_{6} \vee x_{17} \vee x_{19} \wedge x_{7} \vee \neg x_{8} \vee$ $x_{18} \wedge x_{7} \vee x_{10} \vee \neg x_{11} \wedge \neg x_{7} \vee x_{17} \vee \neg x_{19} \wedge \neg x_{7} \vee x_{17} \vee \neg x_{19} \wedge$ $\neg x_{8} \vee x_{9} \vee x_{11} \wedge \neg x_{8} \vee x_{12} \vee \neg x_{20} \wedge x_{8} \vee \neg x_{13} \vee x_{14} \wedge x_{8} \vee \neg x_{13} \vee$ $\neg x_{15} \wedge x_{8} \vee x_{14} \vee \neg x_{15} \wedge x_{8} \vee x_{16} \vee \neg x_{17} \wedge x_{9} \vee \neg x_{12} \vee x_{14} \wedge$ $\neg x_{9} \vee \neg x_{12} \vee x_{16} \wedge \neg x_{9} \vee \neg x_{12} \vee \neg x_{19} \wedge \neg x_{9} \vee x_{15} \vee \neg x_{20} \wedge$ $x_{10} \vee \neg x_{11} \vee x_{17} \wedge \neg x_{10} \vee \neg x_{11} \vee \neg x_{18} \wedge \neg x_{10} \vee \neg x_{12} \vee \neg x_{18} \wedge$ $x_{10} \vee \neg x_{13} \vee x_{18} \wedge x_{10} \vee x_{13} \vee \neg x_{20} \wedge \neg x_{10} \vee x_{17} \vee x_{18} \wedge \neg x_{10} \vee$ $x_{18} \vee \neg x_{19} \wedge x_{11} \vee \neg x_{13} \vee x_{19} \wedge x_{11} \vee x_{14} \vee \neg x_{18} \wedge x_{12} \vee x_{13} \vee$ $\neg x_{18} \wedge x_{13} \vee x_{18} \vee \neg x_{20} \wedge x_{14} \vee \neg x_{19} \vee \neg x_{20} \wedge \neg x_{17} \vee x_{18} \vee x_{20}$.

\section{B. Approximation of $\mathrm{H}^{\gamma}$}

For the calculation of $\beta_{j, \gamma}$ according to (3) we implemented the following procedure, which actually approximates $\beta_{j, \gamma}$ since we employ an approximation of the $\Gamma$-function. We recall that in (3) the (unknown) $N$ is substituted by $M / r$, where $M$ is selected as described in Section IV-A and $r$ is a variable in our calculations.

At first, we represent Eqn. 3 by

$$
\begin{aligned}
\beta_{j, \gamma} & =\frac{M}{r} \cdot\left(\begin{array}{c}
M \\
j
\end{array}\right) \cdot \frac{A_{1}}{A_{2}} \cdot \frac{B_{1}}{B_{2}} \cdot \frac{C_{1}}{C_{2}}, \text { where } \\
A_{1} & =\Gamma\left(a_{1}\right) \text { for } a_{1}=\gamma+j ; \\
A_{2} & =\Gamma\left(a_{2}\right) \text { for } a_{2}=\gamma ; \\
B_{1} & =\Gamma\left(b_{1}\right) \text { for } b_{1}=\gamma \cdot \frac{M}{r} ; \\
B_{2} & =\Gamma\left(b_{2}\right) \text { for } b_{2}=\gamma \cdot\left(\frac{M}{r}-1\right) ; \\
C_{1} & =\Gamma\left(c_{1}\right) \text { for } c_{1}=M-j+\gamma \cdot\left(\frac{M}{r}-1\right) ; \\
C_{2} & =\Gamma\left(c_{2}\right) \text { for } c_{2}=M+\gamma \cdot \frac{M}{r} .
\end{aligned}
$$

Since in our case some of the values are very large, we use intermediately a representation by the natural logarithm, i.e. in the second step we calculate

$$
\begin{aligned}
Z= & \ln \left(\left(\begin{array}{c}
M \\
j
\end{array}\right) \cdot \frac{A_{1}}{A_{2}} \cdot \frac{B_{1}}{B_{2}} \cdot \frac{C_{1}}{C_{2}}\right) \\
= & \ln \left(\begin{array}{c}
M \\
j
\end{array}\right)+\ln A_{1}+\ln B_{1}+\ln C_{1}- \\
& -\ln A_{2}-\ln B_{2}-\ln C_{2} .
\end{aligned}
$$

For each of the $\ln \Gamma(x)$ we employ the following approximation (due to Robert H. Windschitl, 2002):

$$
\begin{aligned}
\ln \Gamma(x) \approx & \frac{1}{2} \cdot(\ln (2 \cdot \pi)-\ln x)+ \\
& +x \cdot\left(-1+\ln \left(x+\frac{1}{12 \cdot x-\frac{1}{10 \cdot x}}\right)\right),
\end{aligned}
$$

i.e. $x=a_{1}, \ldots, c_{2}$. For the binomial coefficient we use the formula

$$
\ln \left(\begin{array}{c}
M \\
j
\end{array}\right)=\sum_{s=1}^{M} \ln s-\sum_{t=1}^{j} \ln t-\sum_{u=1}^{M-j} \ln u .
$$

Finally, we set

$$
\beta_{j, \gamma}=\frac{M}{r} \cdot e^{z} .
$$

Eqn. 20 was then used as a sub-routine in the search for optimum settings of $(r, \gamma)$ :

1) For a fixed $r \geq r_{0}$ we searched for $\gamma$ such that $T_{\gamma}$ from (4) is minimised, i.e. Eqn. 4 and Eqn. 20 were repeatedly calculated for $\gamma \geq \gamma_{0}=0.1$ and $\gamma=\gamma+\delta$, until $T_{\gamma}$ changed only marginally or increased above the minimum value obtained so far.

2) For $r_{0}$ and $r=r+\Delta \leq r_{\max }$, the triplets $\left(r, \gamma, T_{\gamma}\right)$ were recorded and finally $r_{\text {appr }}$ with the minimum value of $T_{\gamma}$ was selected.

3) The output was then determined by $N_{\text {appr }}=M / r_{\text {appr }}$.

\section{Numeric results}

The results are summarised in Table I until Table III. Since both the instances as well as the number of instances are small, the values of $N$ do not provide any statistical information. Our main goal here is to demonstrate that the implementation of the Garnier/Kallel-approach as described in the present section provides approximations $N_{\text {appr }}$ in the region of the exact values $N$.

Since deterministic search is easy to implement and fast, if neighbours can be identified in an easy way, the procedure can be executed for large numbers of $M$, which has been done in the present study, i.e. the $M_{i}$ are relatively large compared to $2^{14}, 2^{20}$ and $2^{21}$, respectively. As a result, we obtained values $\beta_{M}$ that are close or even equal to the corresponding $N$ for $n=14$. We note that for $n=14$ and $M=512$ the value of $N_{\text {appr }}$ is in three out of the five examples equal or close to $N / 2$.

For $n=20$ we see in all five sample functions a clear improvement of the approximation with increasing $M$. The value of $N_{\text {appr }}$ is in four out of the five examples close to $N / 2$ for the largest number $M=2^{14}$

Due to the computational effort caused by the complete search over $\{0,1\}^{21}$, we executed the calculations for three functions only, see Table III. For two out of the three functions we obtain an approximation $N_{\text {appr }}$ in the region of $N / 2$. As for $n=14$ and $n=20$, on the remaining function the approximation procedure returns a value close to $N / 3$.

Since we considered only five (three) functions for each number of variables and even less for different ratios $m / n$, it is not possible to draw any conclusions about the statistics for the number of local maxima. Here, our primary focus is on the quality of approximations $N_{\text {appr }}$ for the exact number of local maxima $N$. 
Future research will focus on a wider range of parameter settings, a larger size of $k$-CNF instances and significantly larger sets of randomly selected $k$-CNF instances.

\begin{tabular}{|r|r|r|rrrrr|}
\hline $\mathrm{m}$ & $\mathrm{N}$ & $\mathrm{M}$ & $\beta_{M}$ & $\gamma\left(r_{\text {appr }}\right)$ & $r_{\text {appr }}$ & $T_{\gamma(r \text { appr }}$ & $N_{\text {appr }}$ \\
\hline 51 & 6 & 128 & 5 & 2.2 & 0.1 & 1.37 & 2 \\
51 & 6 & 256 & 5 & 2.2 & 0.1 & 1.37 & 2 \\
51 & 6 & 512 & 6 & 2.0 & 0.1 & 1.54 & 3 \\
\hline 55 & 13 & 128 & 8 & 2.6 & 0.4 & 81.2 & 3 \\
55 & 13 & 256 & 8 & 2.5 & 0.4 & 90.7 & 3 \\
55 & 13 & 512 & 10 & 2.3 & 0.2 & 308.6 & 4 \\
\hline 59 & 11 & 128 & 6 & 2.8 & 0.1 & 1.35 & 2 \\
59 & 11 & 256 & 8 & 2.4 & 0.3 & 156.0 & 3 \\
59 & 11 & 512 & 8 & 2.1 & 0.2 & 12.8 & 4 \\
\hline 63 & 32 & 128 & 20 & 2.4 & 0.1 & 4859 & 8 \\
63 & 32 & 256 & 24 & 2.3 & 0.1 & 9185 & 10 \\
63 & 32 & 512 & 27 & 2.2 & 0.1 & 17574 & 12 \\
\hline 68 & 10 & 128 & 6 & 2.7 & 0.4 & 14.3 & 2 \\
68 & 10 & 256 & 10 & 2 & 0.1 & 2.1 & 5 \\
68 & 10 & 512 & 9 & 1.7 & 0.2 & 23.6 & 5 \\
\hline
\end{tabular}

TABLE I

RESULTS FOR $n=14, k=3$

\begin{tabular}{|r|r|c|rrrrr|}
\hline $\mathrm{m}$ & $\mathrm{N}$ & $\mathrm{M}$ & $\beta_{M}$ & $\gamma\left(r_{\text {appr }}\right)$ & $r_{\text {appr }}$ & $T_{\gamma(r \text { appr })}$ & $N_{\text {appr }}$ \\
\hline 78 & 213 & $2^{10}$ & 66 & 0.1 & 4.23 & $1 \times 10^{6}$ & 16 \\
78 & 213 & $2^{12}$ & 112 & 0.1 & 2.90 & $2 \times 10^{9}$ & 39 \\
78 & 213 & $2^{14}$ & 157 & 0.1 & 2.36 & $1 \times 10^{14}$ & 67 \\
\hline 82 & 35 & $2^{10}$ & 19 & 0.1 & 2.64 & 321.39 & 7 \\
82 & 35 & $2^{12}$ & 32 & 0.1 & 2.09 & 5.06 & 15 \\
82 & 35 & $2^{14}$ & 33 & 0.1 & 2.06 & 2139.39 & 16 \\
\hline 86 & 142 & $2^{10}$ & 72 & 0.1 & 2.97 & $6 \times 10^{6}$ & 24 \\
86 & 142 & $2^{12}$ & 117 & 0.1 & 2.21 & $2 \times 10^{11}$ & 53 \\
86 & 142 & $2^{14}$ & 136 & 0.1 & 2.04 & $6 \times 10^{13}$ & 67 \\
\hline 90 & 33 & $2^{10}$ & 21 & 0.1 & 2.57 & 1012.02 & 8 \\
90 & 33 & $2^{12}$ & 31 & 0.1 & 2.06 & 134.94 & 15 \\
90 & 33 & $2^{14}$ & 33 & 0.1 & 2.00 & 2723 & 17 \\
\hline 94 & 15 & $2^{10}$ & 5 & 5.1 & 3.10 & 5.52 & 2 \\
94 & 15 & $2^{12}$ & 13 & 0.1 & 2.16 & 27.2 & 6 \\
94 & 15 & $2^{14}$ & 15 & 0.1 & 2.00 & 109.57 & 8 \\
\hline
\end{tabular}

TABLE II

RESULTS FOR $n=20, k=3$

\begin{tabular}{|c|r|c|rrrrr|}
\hline $\mathrm{m}$ & $\mathrm{N}$ & $\mathrm{M}$ & $\beta_{M}$ & $\gamma\left(r_{\text {appr }}\right)$ & $r_{\text {appr }}$ & $T_{\gamma\left(r_{\text {appr }}\right)}$ & $N_{\text {appr }}$ \\
\hline 79 & 109 & $2^{13}$ & 62 & 0.1 & 2.8 & 588792 & 23 \\
79 & 109 & $2^{14}$ & 72 & 0.1 & 2.5 & 4927846 & 29 \\
79 & 109 & $2^{15}$ & 75 & 0.1 & 2.5 & 7841494 & 31 \\
\hline 89 & 39 & $2^{13}$ & 25 & 0.1 & 2.6 & 804 & 10 \\
89 & 39 & $2^{14}$ & 26 & 0.1 & 2.5 & 2811 & 10 \\
89 & 39 & $2^{15}$ & 37 & 0.1 & 2.1 & 35696 & 18 \\
\hline 99 & 47 & $2^{13}$ & 44 & 0.1 & 2.1 & 251220 & 21 \\
99 & 47 & $2^{14}$ & 43 & 0.1 & 2.1 & 213092 & 21 \\
99 & 47 & $2^{15}$ & 45 & 0.1 & 2.0 & 698186 & 22 \\
\hline
\end{tabular}

TABLE III

RESULTS FOR $n=21, k=3$

\section{LOCAL MAXIMA AND $k$-CNF}

For an arbitrary $\tilde{\sigma} \in\{0,1\}^{n}$ and $F \in \mathcal{F}_{k}(n, m)$, we set $\mathcal{C}_{0}(F, \tilde{\sigma})=\{C \mid C \in \mathcal{C}(F) \wedge C(\tilde{\sigma})=0\}$ and $\mathcal{C}_{1}(F, \tilde{\sigma})=\{C \mid C \in$ $\mathcal{C}(F) \wedge C(\tilde{\sigma})=1\}$. Thus, clauses from $\mathcal{C}_{1}(F, \tilde{\sigma})$ have at least one literal among the $k$ literals that returns 1 on $\tilde{\sigma}$. Since in (1) we have $d\left(\tilde{\sigma}, \tilde{\sigma}^{\prime}\right)=1$, clauses with at least two literals returning 1 on $\tilde{\sigma}$ do not affect the re-calculation of $Z_{F}$ in neighbourhood transitions out of $\tilde{\sigma}$. We therefore partition $\mathcal{C}_{1}(F, \tilde{\sigma})$ into $\mathcal{C}_{1}^{(1)}(F, \tilde{\sigma})$ and $\mathcal{C}_{\mathbf{1}}^{(\geq 2)}(F, \tilde{\sigma})$, i.e. $\mathcal{C}_{\mathbf{1}}^{(1)}(F, \tilde{\sigma})$ contains all $C \in \mathcal{C}(F)$ with exactly one literal that returns 1 on $\tilde{\sigma}$. We note the following simple observation:

Lemma 1: The truth assignment $\tilde{\sigma}$ is a local maximum in $\mathcal{L}(F)$ iff for all $\tilde{\sigma}^{\prime} \in \mathcal{N}(\tilde{\sigma})$ :

$$
\begin{aligned}
\left|\left\{C \mid C\left(\tilde{\sigma}^{\prime}\right)=1 \wedge C \in \mathcal{C}_{\mathbf{0}}(F, \tilde{\sigma})\right\}\right| \\
\leq \quad\left|\left\{C \mid C\left(\tilde{\sigma}^{\prime}\right)=0 \wedge C \in \mathcal{C}_{\mathbf{1}}^{(1)}(F, \tilde{\sigma})\right\}\right| .
\end{aligned}
$$

Here, we do not exclude $Z_{F}(\tilde{\sigma})=m$.

For a literal $x^{\eta}$ we use $x^{\eta} \in C$ to express that $x^{\eta}$ is part of the disjunctive term $C$. Let $X_{\mathbf{0}}(\tilde{\sigma})=\left\{x \mid \exists C \in \mathcal{C}_{\mathbf{0}}(F, \tilde{\sigma}) \wedge x^{\bar{\sigma}} \in C\right\} \mid$ and $p=\left|X_{\mathbf{0}}(\tilde{\sigma})\right|$ be the number of variables that occur in clauses of $\mathcal{C}_{0}(F, \tilde{\sigma})$, where we employ $\sigma^{\bar{\sigma}} \equiv 0$. Furthermore, we set $q=$ $\left|\mathcal{C}_{\mathbf{0}}(F, \tilde{\sigma})\right|, r=\left|\mathcal{C}_{\mathbf{1}}^{(1)}(F, \tilde{\sigma})\right|$, and $s=\left|\mathcal{C}_{\mathbf{1}}^{(\geq 2)}(F, \tilde{\sigma})\right|$. Thus, we have for $F \in \mathcal{F}_{k}(n, m)$

$$
m=q+r+s .
$$

For $X_{1}=\left\{x \mid \exists C \in \mathcal{C}_{1}^{(1)}(F, \tilde{\sigma}) \wedge x^{\sigma} \in C\right\}, t=\left|X_{1}\right|$, and $h_{u}=$ $\left|\left\{C \mid C \in \mathcal{C}_{\mathbf{1}}^{(1)}(F, \tilde{\sigma}) \wedge x_{i_{u}}^{\sigma_{i u}} \in C\right\}\right|$ we have

$$
\sum_{u=1}^{t} h_{u}=r
$$

since the corresponding subsets of clauses have to be disjoint (otherwise, a clause from the intersection would belong to $\mathcal{C}_{1}^{(\geq 2)}(F, \tilde{\sigma})$ ).

Lemma 2: If $x_{i_{u}} \in X_{\mathbf{1}} \backslash X_{\mathbf{0}} \neq \emptyset$, then the neighbourhood transition that involves $x_{i_{u}}$ diminishes $Z_{F}(\tilde{\sigma})$ by $h_{u}$.

This follows from the definitions of $\mathcal{C}_{\mathbf{0}}(F, \tilde{\sigma})$ and $\mathcal{C}_{\mathbf{1}}^{(1)}(F, \tilde{\sigma})$. For $f_{u}=\left|\left\{C \mid C \in \mathcal{C}_{\mathbf{0}}(F, \tilde{\sigma}) \wedge x_{i_{u}}^{\overline{\sigma_{i u}}} \in C\right\}\right|$, Lemma 1 can now be rewritten as

Lemma 3: The truth assignment $\tilde{\sigma}$ is a local maximum in $\mathcal{L}(F)$ iff $X_{0} \subseteq X_{1}$ and for $x_{i_{u}} \in X_{0}$ :

$$
f_{u} \leq h_{u}
$$

We note that by definition

$$
\sum_{u=1}^{p} f_{u}=q \cdot k
$$

and (24) and (25) imply for a local maximum

$$
q \cdot k \leq r .
$$

Let $\mathcal{M}_{k}^{\tilde{\sigma}}(n, m) \subseteq \mathcal{F}_{k}(n, m)$ denote the set of $k$-CNF that have $\tilde{\sigma}$ as a local maximum for the neighbourhood defined by $\mathcal{N}(\tilde{\sigma})$ and the objective function defined by $Z_{F}$, where we require $Z_{F}(\tilde{\sigma})<m$, i.e. $q \geq 1$ and $\tilde{\sigma}$ is not a satisfying assignment.

We are now going to derive a (rough) upper bound for $M_{\tilde{\sigma}}=$ $\left|\mathcal{M}_{k}^{\tilde{\sigma}}(n, m)\right|$. As will be seen later, the ratio $2^{n} \cdot M_{\tilde{\sigma}} /\left|\mathcal{F}_{k}(n, m)\right|$, when approximated by using an upper bound of $M_{\tilde{\sigma}}$, then provides some information about typical values for the number of local maxima for $k$-CNF in terms of parameters $(k, n, m)$.

For fixed $(p, q, r, s)$, we consider the number of potential sets $\mathcal{C}_{0}(F, \tilde{\sigma}), \mathcal{C}_{1}^{(1)}(F, \tilde{\sigma})$, and $\mathcal{C}_{1}^{(2)}(F, \tilde{\sigma})$ under the assumption that the fixed truth assignment $\tilde{\sigma}$ is a local maximum. Here, it is useful to consider bipartite graphs where one set of nodes represents the clauses of $\mathcal{C}$ with fixed degree $k$, and the other set of nodes is formed by the elements of $\left\{\sigma_{i} ; i=1,2, \ldots, n\right\}$.

For $\mathcal{C}_{0}(F, \tilde{\sigma})$ we have to ensure that each of the $p$ elements of $X_{\mathbf{0}}(\tilde{\sigma})$ is present in at least one of the clauses from $\mathcal{C}_{\mathbf{0}}$ and we therefore need

$$
q \cdot k \geq p \geq k \text { and }\left(\begin{array}{l}
p \\
k
\end{array}\right) \geq q .
$$


Let $A(p, q, k)$ denote the number of sets $\mathcal{H}$ of size $q$ consisting of $k$-selections $S=\left\{x_{i_{1}}, \ldots, x_{i_{k}}\right\}$ out of $p$ variables of $X_{\mathbf{0}}(\tilde{\sigma})$ such that $\forall x\left(x \in X_{\mathbf{0}} \rightarrow \exists S(S \in \mathcal{H} \wedge x \in S)\right)$. Since in the given context the elements of $X_{\mathbf{0}}(\tilde{\sigma})$ are independent of each other, we have

$$
\begin{aligned}
A(p, q, k)= & \left(\begin{array}{c}
p \\
k \\
q
\end{array}\right)-p \cdot A(p-1, q, k)- \\
& -\left(\begin{array}{c}
p \\
2
\end{array}\right) \cdot A(p-2, q, k)-\ldots- \\
& -\left(\begin{array}{c}
p \\
s_{\text {fin }}
\end{array}\right) \cdot A\left(p-s_{\text {fin }}, q, k\right),
\end{aligned}
$$

where $s_{\text {fin }}$ is defined by $\left(\begin{array}{c}p-\left(s_{\text {fin }}+1\right) \\ k\end{array}\right)<q$. By substituting the $A(p-$ $i, q, k)$ recurrently, we obtain the inclusion/exclusion-type equation

$$
A(p, q, k)=\sum_{i=0}^{s_{\text {fin }}}(-1)^{i} \cdot\left(\begin{array}{c}
p \\
i
\end{array}\right) \cdot\left(\begin{array}{c}
p-i \\
k \\
q
\end{array}\right),
$$

which represents the number of sets $\mathcal{C}_{\mathbf{0}}(F, \tilde{\sigma})$. Taking $\left(\begin{array}{c}p \\ 2 \cdot s-1\end{array}\right)$. $\left(\begin{array}{c}\left(\begin{array}{c}p-2 \cdot s-1 \\ k \\ q\end{array}\right)\end{array}\right)-\left(\begin{array}{c}p \\ 2 \cdot s\end{array}\right) \cdot\left(\begin{array}{c}\left(\begin{array}{c}p-2 \cdot s \\ k\end{array}\right) \\ q\end{array}\right)$ together and applying Stirling's formula, one can see that the impact of $\sum_{i=1}^{s}(-1)^{i} \cdot\left(\begin{array}{c}p \\ i\end{array}\right) \cdot\left(\begin{array}{c}p-i \\ k\end{array}\right)$ is only marginal and we therefore employ $\left(\begin{array}{c}p \\ k \\ q\end{array}\right)$ to upper bound the number of sets $\mathcal{C}_{\mathbf{0}}(F, \tilde{\sigma})$.

For $\mathcal{C}_{1}^{(1)}(F, \tilde{\sigma})$ we consider the set $X_{\mathbf{1}}$ : for $f_{u}$ clauses from $\mathcal{C}_{0}(F, \tilde{\sigma})$ with $x_{i_{u}}^{\overline{\sigma_{i}}}$ we have $h_{u} \geq f_{u}$ clauses from $\mathcal{C}_{\mathbf{1}}^{(1)}(F, \tilde{\sigma})$ with $x_{i_{u}}^{\sigma_{i_{u}}}$, if $\tilde{\sigma}$ is a local maximum. In each of the $h_{u}$ clauses, the literals different from $x_{i_{u}}^{\sigma_{i}}$ are of the type $x_{j}^{\overline{\sigma_{j}}}$ due to the definition of $\mathcal{C}_{\mathbf{1}}^{(1)}(F, \tilde{\sigma})$. Thus, the number of different sets $\mathcal{C}_{\mathbf{1}}^{(1)}(F, \tilde{\sigma})$ can be upper bounded by

$$
\sum_{t=p}^{n}\left(\begin{array}{c}
n-p \\
t-p
\end{array}\right) \sum_{\substack{\left(h_{1}, \ldots, h_{t}\right) \\
h_{1} \geq f_{1}, \ldots, h_{p} \geq f_{p}}} \prod_{u=1}^{t}\left(\begin{array}{c}
\left(\begin{array}{c}
n-1 \\
k-1
\end{array}\right) \\
h_{u}
\end{array}\right) .
$$

We recall that $t \geq p$ is required by Lemma 1 .

For $\mathcal{C}_{\mathbf{1}}^{(\geq 2)}(F, \tilde{\sigma})$ we consider the set of all $\left(\begin{array}{l}n \\ k\end{array}\right) \cdot 2^{k}$ clauses: since $\tilde{\sigma}$ is fixed, among the set of all clauses there are $\left(\begin{array}{l}n \\ k\end{array}\right)$ clauses that return 0 on $\tilde{\sigma}$ (the clauses of $\mathcal{C}_{0}(F, \tilde{\sigma})$ are drawn from this subset); there are $\left(\begin{array}{l}n \\ k\end{array}\right) \cdot k$ clauses with excatly one literal of type $x_{i_{u}}^{\sigma_{i}}$ (the clauses of $\mathcal{C}_{\mathbf{1}}^{(1)}(F, \tilde{\sigma})$ are drawn from this subset). Thus, the number of different sets $\mathcal{C}_{\mathbf{1}}^{(\geq 2)}(F, \tilde{\sigma})$ can be upper bounded by

$$
\left(\begin{array}{c}
\left(\begin{array}{l}
n \\
k
\end{array}\right) \cdot\left(2^{k}-k-1\right) \\
s
\end{array}\right) .
$$

We assume at first $t=n$ (implicitly also $q \geq n / k$ ) and set $r=q \cdot k+\Delta$ for $\Delta \geq 0$, cf. (27). Based on $\left(\begin{array}{c}K \\ a\end{array}\right) \cdot\left(\begin{array}{c}K \\ b\end{array}\right) \geq\left(\begin{array}{c}K \\ a+b\end{array}\right)$ (and the remark after (30)), we summarize (30), (31) and (32) to

$$
\begin{aligned}
& M_{\tilde{\sigma}}<\sum_{p=k}^{n}\left(\begin{array}{l}
n \\
p
\end{array}\right) \cdot\left\{\sum_{\substack{q+r+s=m \\
r \geq k \cdot q, q \geq 1}}\left(\begin{array}{c}
p \\
k
\end{array}\right)\right) \cdot\left(\sum_{t=p}^{n}\left(\begin{array}{c}
n-p \\
t-p
\end{array}\right) \times\right.
\end{aligned}
$$

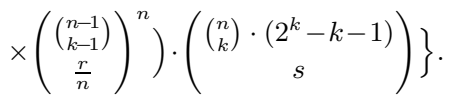

(Note: we use $\left(\begin{array}{c}A \\ B\end{array}\right)^{n}$ for $\left\{\left(\begin{array}{l}A \\ B\end{array}\right)\right\}^{n}$.

We are now going to identify $(q, r, s)$ such that the product on the RHS of (33) is maximised for fixed $(p, k, n, m)$. At first, we consider for variable $r$ and $s$ the product

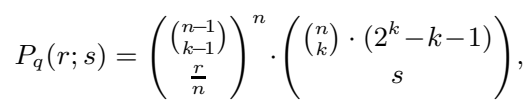

where $r+s=m-q$. We analyse $P_{q}(r ; s) \leq P_{q}(r-1 ; s+1)$, which for $r=a \cdot n+b, 1 \leq b<n$, turns to

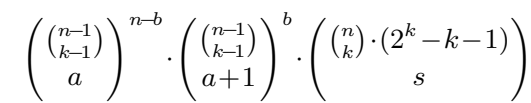

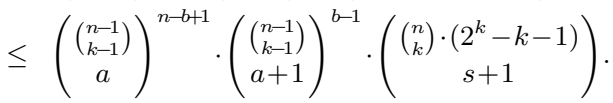

For $b=0$ we take $a-1$ and $b^{\prime}=n$. By straightforward calculations one obtains that (35) is valid if $r \geq \widehat{r}$ for

$$
\widehat{r}=\frac{(m-q) \cdot\left(k+\varepsilon_{1}\right)-(n-b) \cdot\left(2^{k}-k-1\right)+k+\varepsilon_{2}}{2^{k}-1+\varepsilon_{3}},
$$

where $\varepsilon_{1}=n /\left(\begin{array}{l}n \\ k\end{array}\right), \varepsilon_{2}=b /\left(\begin{array}{l}n \\ k\end{array}\right)$, and $\varepsilon_{3}=(n+1) /\left(\begin{array}{l}n \\ k\end{array}\right)$. Here, we assume that $m$ is sufficiently large in relation to $n$ and $2^{k}$, which will be discussed further below in more detail.

Thus, if we assume $\widehat{r}>k \cdot q$ (cf. (27)), then (34) increases for increasing $s$ from $0 \leq s \leq \widehat{s}$ and (34) decreases for increasing $s$ from $\widehat{s}<s \leq s_{\max }$, where $\widehat{s}=m-q-\widehat{r}$ and $s_{\max }=m-q-k \cdot q$.

The condition $\widehat{r} \geq k \cdot q$ results in an upper bound for $q$ :

$$
\begin{aligned}
k \cdot q & \leq \frac{(m-q) \cdot\left(k+\varepsilon_{1}\right)-(n-b) \cdot\left(2^{k}-k-1\right)+k+\varepsilon_{2}}{2^{k}-1+\varepsilon_{3}} \\
q & \leq q_{1}=\frac{m \cdot\left(k+\varepsilon_{1}\right)-(n-b) \cdot\left(2^{k}-k-1\right)+k+\varepsilon_{2}}{k \cdot\left(2^{k}+\varepsilon_{4}\right)}
\end{aligned}
$$

where $\varepsilon_{4}=(n+n / k+1) /\left(\begin{array}{l}n \\ k\end{array}\right)$. Furthermore, we need $k \cdot q / n \leq$ $r / n<\left(\begin{array}{l}n-1 \\ k-1\end{array}\right)$ and $k \cdot q \leq r=m-q-s \leq m-q$, which leads to

$$
\begin{aligned}
& q<q_{2}=\left(\begin{array}{l}
n \\
k
\end{array}\right), \\
& q \leq q_{3}=\frac{m}{k+1} .
\end{aligned}
$$

Summarizing these observations, we obtain that $P_{q}(r ; s)$ from (34) is maximized (ignoring integer representations) at $P_{q}(\widetilde{r} ; \widetilde{s})$ for $\widetilde{s}=m-q-\widetilde{r}$ with $\widetilde{r}=\max \{k \cdot q, \widehat{r}\}$, where $q$ is fixed but must obey the minimum upper bound defined by (37) until (39), depending upon the value of $\widetilde{r}$.

So far, we kept the parameter $q$ fixed. Now we take into account $\left(\begin{array}{c}\left(\begin{array}{c}p \\ k \\ q\end{array}\right)\end{array}\right)$ from (33) for $p=n$ and try to maximize (and to compare)

$$
\begin{aligned}
& \left(\begin{array}{c}
\left(\begin{array}{c}
n \\
k
\end{array}\right) \\
q
\end{array}\right) \cdot P_{q}(k \cdot q ; m-q-k \cdot q) \\
& \left(\begin{array}{c}
\left(\begin{array}{c}
n \\
k
\end{array}\right) \\
q
\end{array}\right) \cdot P_{q}(\widehat{r} ; m-q-\widehat{r}) .
\end{aligned}
$$

By using a representation similar to the one of (35) and setting $A=\left(\begin{array}{l}n \\ k\end{array}\right) \cdot\left(2^{k}-k-1\right), B=\left(\begin{array}{l}n \\ k\end{array}\right)$, the assumption about an increasing value of (40) for increasing $q$ leads to

$$
\left(\frac{A-m+(k+1) \cdot q+1}{m-(k+1) \cdot q-k}\right)^{k+1}<\left(\frac{B-q+b / k}{q+(n-b) / k}\right)^{k} \cdot \frac{B-q}{q+1} .
$$

Depending on the value of $b$, one has to consider two cases of the type

$$
\frac{A-m+(k+1) \cdot q+1}{m-(k+1) \cdot q-k}<\frac{B-q}{q+1}
$$


The resulting upper bound

$$
q<\frac{m-2^{k}+1+(m-1) /\left(\begin{array}{l}
n \\
k
\end{array}\right)}{2^{k}+2 /\left(\begin{array}{l}
n \\
k
\end{array}\right)}
$$

is similar to the upper bound (37). Based on these observations we conjecture that (40) and (41) are maximized for $q^{*} \sim m / 2^{k}$ and $\widehat{r} \lesssim k \cdot q^{*}$. A detailed analysis of all sub-cases will be subject of further research.

We note that $q^{*} \sim m / 2^{k}$ and $\widehat{r} \lesssim k \cdot q^{*}$ actually ignore the relation of $m$ to $n$ and $k$. A more detailed analysis of (37) shows that $q_{1} \sim m / 2^{k}-(n-b) / k$, where $1 \leq b<n$. If $m \lesssim(n-b) \cdot \frac{2^{k}}{k}$ and $1<<b<n$, i.e. $m$ is in the region of the phase transition threshold [1], then $q_{1}<0$ and, moreover, for $q$ from (44) the condition $q>\lceil n / k\rceil$ might no longer be valid, which is required by (26) for $p=n$. If this is the case, the maximum value of (40) changes significantly. Therefore, we conjecture that for the neighbourhood under consideration and $m / n$ in the region of

$$
\frac{m}{n} \leq O\left(\frac{2^{k}}{k}\right)
$$

(which differs roughly by $k$ from the phase transition threshold) the value of $M_{\tilde{\sigma}}$ is (for a large fraction of $\tilde{\sigma}$ ) significantly smaller than for $m / n>>O\left(2^{k} / k\right)$. A detailed analysis requires a careful consideration of sub-cases for $p<n$ and (33).

In Lemma 3, (30), (31), and (32) we exploit only information about $x_{i}^{\overline{\sigma_{i}}}$ vs. $x_{i}^{\sigma_{i}}$, i.e. information about the actual values of $\sigma_{i}$ has no impact on $M_{\tilde{\sigma}}$ at all. Thus, $M_{\tilde{\sigma}}$ depends on structural parameters $(n, k, m)$ only:

Lemma 4: If $\tilde{\sigma}, \tilde{\eta} \in\{0,1\}^{n}$, then $M_{\tilde{\sigma}}=M_{\tilde{\eta}}$ for a given class $\mathcal{F}_{k}(n, m)$

Given $(n, k, m)$, we denote by $R\left(n, k, m, q^{*}\right)$ the maximum value of $\left(\begin{array}{c}n \\ k\end{array}\right) \cdot P_{q}(r ; m-q-r)$ as presented in (40) and (41). In (33), the value of $\sum_{p=k}^{n}\left(\begin{array}{l}n \\ p\end{array}\right) \sum_{t=p}^{n}\left(\begin{array}{c}n-p \\ t-p\end{array}\right)$ can be upper bounded by $3^{n}$. We now have for $m \leq\left(\begin{array}{l}n \\ k\end{array}\right) \cdot\left(2^{k}-k-1\right)$ the upper bound

$$
M_{\tilde{\sigma}}<(n-k)^{2} \cdot \frac{m^{2}}{2 \cdot(k+1)} \cdot 3^{n} \cdot R\left(n, k, m, q^{*}\right),
$$

where $R\left(n, k, m, q^{*}\right)$ depends on the relation of $m$ to $n$ and $k$. For an upper bound of the average number of local maxima per $k$-CNF, one has to multiply the RHS of (46) by $2^{n}$ (cf. Lemma 4) and to divide the expression by the number of $k$-CNF of length $m$. A rough estimate for $R\left(n, k, m, m / 2^{k}\right)$, i.e. by using Stirling's formula as well as $q^{*} \sim m / 2^{k}$ and $r \sim k \cdot q^{*}$, indicates that simply taking $q^{*} \sim m / 2^{k}$ is insufficient to obtain a proper upper bound. In order to compensate the first three factors on the RHS of (46), the tighter upper bound for $q^{*}$ from (44) has to be employed rather than an asymptotic approximation. Thus, to obtain asymptotic expressions for the average number of local maxima per $k$-CNF requires a detailed analysis of $R\left(n, k, m, q^{*}\right)$ for tight upper bounds of $q^{*}$, which will be subject of further research.

\section{CONCLUding REMARKS}

The Garnier/Kallel-approach requires a partition of the search space into attraction basins, i.e. within each neighbourhood a single element with the maximum value of the objective function is assumed. This assumption does not apply to the neighbourhood in our study. Nevertheless, our computational experiments provide evidence that the sampling-based method for the approximation of the number of local maxima seems to work in the context of $k$-SAT instances. In seven out of the ten instances analysed the approximation $N_{\text {appr }}$ comes close or is equal to $N / 2$; for the remaining three instances the approximations are in the region of $N / 3$. The approximations steadily improve with the increase of the size of sampling information. Furthermore, the outline of our method for obtaining upper bounds for the average number of local maxima per $k$-SAT instance suggests that the magnitude of the fraction of local maxima relative to the total number of truth assignments changes around $\Theta\left(2^{k} / k\right)$, which is close to the phase transition threshold $2^{k} \cdot \log 2-O(k)$. The expression $\Theta\left(2^{k} / k\right)$ is, of course, dependent on the specific neighbourhood underlying our calculations. We intend to analyse a variety of neighbourhood relations proposed in the literature [8], [20], [21], where it would be interesting to find out if the average number of local maxima (or how close the "breaking point" comes to the phase transition threshold) can be related to the quality of the associated local search procedure. Future research will also include a more comprehensive analysis of the Garnier/Kallel-approach for larger $k$-SAT instances and larger sets of parameter settings. Furthermore, we intend to apply the Garnier/Kallel-method in a completely different context, namely the simulation of protein folding in various lattice models and for different types of the objective function. Protein folding simulation in the HP-model is NP-complete and population-based heuristics are an obvious choice to tackle the folding problem [7]. The standard method for identifying local minima in folding landscapes are barrier trees [26]. As pointed out by Garnier and Kallel [5], “... from a practical point of view, the tree describing the repartition of local optima is unknown and too expensive in terms of computational cost to determine for a given landscape." Thus, approximations as described in the present paper might be helpful for the analysis of energy landscapes induced by protein sequences. However, a major obstacle seems to be the selection of a suitable neighbourhood relation that complies with the underlying energy function and allows reversible neighbourhood transitions.

\section{REFERENCES}

[1] D. Achlioptas and Y. Peres, "The threshold for random k-SAT is $2^{k}$. $\log 2-O(k)$, , Journal of the American Mathematical Society, vol. 17 , no. 4, pp. 947-973, 2004.

[2] A.A. Albrecht, "A stopping criterion for logarithmic simulated annealing," Computing, vol. 78, no. 1, pp. 55-79, 2006.

[3] T. Brueggemann and W. Kern, "An improved local search algorithm for 3-SAT," Theoretical Computer Science, vol. 329, no. 1-3, pp. 303313, 2004.

[4] E. Dantsin and A. Wolpert, "An improved upper bound for SAT," In Proceedings SAT 2005, LNCS vol. 3569, pp. 400-407, Heidelberg: Springer-Verlag, 2005.

[5] J. Garnier and L. Kallel, "Efficiency of local search with multiple local optima," SIAM Journal on Discrete Mathematics, vol. 15, no. 1 , pp. $122-141,2002$

[6] A. Gerevini and I. Serina, "Planning as propositional CSP: From WalkSAT to local search techniques for action graphs," Constraints, vol. 8, no. 4, pp. 389-413, 2003.

[7] H.J. Greenberg, W.E. Hart, G. Lancia, "Opportunities for combinatorial optimization in computational biology," INFORMS Journal on Computing, vol. 16, pp. 211-231, 2004.

[8] H.H. Hoos and Th. Stützle, "Towards a characterisation of the behaviour of stochastic local search algorithms for SAT," Artificial Intelligence, vol. 112, pp. 213-232, 1999.

[9] O.C. Martin, R. Monasson and R. Zecchina, "Statistical mechanics methods and phase transitions in optimization problems," Theoretical Computer Science, vol. 265, pp. 3-67, 2001.

[10] M. Mézard and R. Zecchina, "Random $K$-satisfiability problem: from an analytic solution to an efficient algorithm," Physical Review E, vol. 66, pp. 056126-1-27, 2002.

[11] D. Mitchell, B. Selman and H. Levesque, "Hard and easy distributions of SAT problems," In Proceedings of the $10^{\text {th }}$ National Conference on Artificial Intelligence, pp. 459-465, Menlo Park, CA: AAAI Press, 1992.

[12] R. Monasson and R. Zecchina, "Statistical mechanics of the random K-SAT problem,” Physical Review E, vol. 56, pp. 1357-1361, 1997.

[13] A. Montanari, G. Parisi and F. Ricci-Tersenghi, "Instability of one-step replica-symmetry-broken phase in satisfiability problems," Journal of Physics A: Mathematics and General, vol. 37, pp. 2073-2091, 2004. 
[14] R. Paturi, P. Pudlák, M.E. Saks and F. Zane, "An improved exponential-time algorithm for $k$-SAT," Journal of the ACM, vol. 52 , no. 3, pp. 337-364, 2005.

[15] C.R. Reeves and A.V. Eremeev, "Statistical analysis of local search landscapes," Journal of the Operational Research Society, vol. 55, pp. 687-693, 2004.

[16] Ch.M. Reidys and P.F. Stadler, "Combinatorial landscapes," SIAM Review, vol. 44, no. 1, pp. 3-54, 2002.

[17] U. Schöning, "A probabilistic algorithm for $k$-SAT based on limited local search and restart," Algorithmica, vol. 32, no. 4, pp. 615-623, 2002.

[18] R. Schuler, "An algorithm for the satisfiability problem of formulas in conjunctive normal form," Journal of Algorithms, vol. 54, no. 1 , pp. 40-44, 2005.

[19] D. Schuurmans and F. Southey, "Local search characteristics of incomplete SAT procedures," Artificial Intelligence, vol. 132, pp. 121150, 2001.

[20] S. Seitz, M. Alava and P. Orponen, "Threshold behaviour of WalkSAT and focused Metropolis search on random 3-satisfiability," In Proceedings SAT 2005, LNCS vol. 3569, pp. 475-481, Heidelberg: Springer-Verlag, 2005.

[21] S. Seitz and P. Orponen, "An efficient local search method for random 3-satisfiability," Electronic Notes in Discrete Mathematics, vol. 16 , pp. 71-79, 2003.
[22] B. Selman, H.A. Kautz and B. Cohen, "Noise strategies for improving local search," In Proceedings AAAI-94, pp. 337-343, Cambridge, MA: MIT Press, 1994.

[23] B. Selman, H. Levesque and D. Mitchell, "A new method for solving hard satisfiability problems," In Proceedings AAAI-92, pp. 440-446, Cambridge, MA: MIT Press, 1992.

[24] K. Steinhöfel, A. Skaliotis and A.A. Albrecht, "Relating time complexity of protein folding simulation to approximations of folding time," Computer Physics Communications, vol. 176, no. 7, pp. 165$170,2007$.

[25] K. Steinhöfel, A. Skaliotis and A.A. Albrecht, "Stochastic protein folding simulation in the d-dimensional HP-model," In Proceedings Bioinformatics Research and Development (BIRD'07), LNBI vol. 4414, pp. 381-394, Heidelberg: Springer-Verlag, 2007.

[26] D. Wales, "Energy landscapes", Cambridge: Cambridge University Press, 2003

[27] W. Zhang, "Configuration landscape analysis and backbone guided local search. Part I: Satisfiability and maximum satisfiability," Artificial Intelligence, vol. 158, pp. 1-26, 2004 